\title{
Renal growth in isolated methylmalonic acidemia
}

\author{
Paul S. Kruszka, MD1, Irini Manoli, MD, PhD'1, Jennifer L. Sloan, PhD, MS', Jeffrey B. Kopp, MD², and \\ Charles P. Venditti, MD, PhD ${ }^{1}$
}

Purpose: We sought to predict renal growth based on clinical and metabolic parameters in patients with isolated methylmalonic acidemia, a group of disorders associated with chronic kidney disease.

Methods: Fifty patients with methylmalonic acidemia, followed from 2004 to 2011, were classified by molecular genetics and studied using a combined cross-sectional and longitudinal design that included renal ultrasound examinations, anthropometric measurements, and metabolic phenotyping. Renal length was compared with that of healthy controls and modeled to other clinical parameters using multiple-regression analyses.

Results: Comparisons with age-matched controls showed that renal length in subjects with methylmalonic acidemia was significantly decreased $(P<0.05)$. Stepwise regression modeling found that combinations of height, serum cystatin $\mathrm{C}$, and serum methymalonic acid concentrations best predicted kidney size. The regression equations used to generate methylmalonic acidemia kidney nomograms were renal length $(\mathrm{cm})=6.79+0.22 \times$ age for the controls and $6.80+0.09$ $\times$ age for the methylmalonic acidemia cohort $(P<0.001$; constant and slope).

Conclusion: Renal length, reflective of kidney growth, significantly decreased in patients with methylmalonic acidemia over time as compared with controls and was predictable with select clinical parameters. Cystatin $\mathrm{C}$ and serum methylmalonic acid concentrations were highly correlated with smaller kidneys and decreased renal function in this patient population.

Genet Med advance online publication 2 May 2013

Key Words: chronic kidney disease; cystatin C; isolated methylmalonic acidemia; methylmalonyl-CoA mutase; renal growth; renal ultrasound; vitamin B12
Methylmalonic acidemia (MMA) is an inborn error of metabolism caused by deficiency of the mitochondrial enzyme, methylmalonyl-CoA mutase $(M U T)$. This vitamin B12dependent enzyme catalyzes the isomerization of L-methylmalonyl-CoA into succinyl-CoA at the terminal step of propionyl-CoA oxidation. ${ }^{1}$ Isolated MMA exhibits genetic heterogeneity and most commonly is caused by mutations in the MUT gene, resulting in either complete $\left(m u t^{0}\right)$ or partial $\left(m u t^{-}\right)$loss of enzymatic activity, which can also be caused by cblA (MMAA), cblB (MMAB), and the much rarer cblD variant 2 (MMADHC) complementation classes. The latter forms of MMA are the result of enzymopathies that impair the intracellular transport and metabolism of cobalamin. ${ }^{1}$ Unlike mut MMA, most patients with $c b l A$ and $50 \%$ of $c b l B$ forms of MMA are responsive to parenteral vitamin B12, a clinical and biochemical determinant that has been associated with improved survival and milder disease. ${ }^{2-7}$

MMA patients exhibit a well-recognized phenotype that features massive elevations of disease-related metabolites in the body fluids and multisystemic complications such as growth impairment, pancreatitis, metabolic stroke of the basal ganglia, intellectual disability, and end-stage kidney disease. ${ }^{1}$ Although this disorder has historically had a poor prognosis, with most vitamin B12-nonresponsive patients dying in the first decade of life, ${ }^{2-4}$ earlier diagnosis through newborn screening and more aggressive treatment has afforded increased survival. ${ }^{7,8}$
This highlights the need to understand the natural history of MMA and to develop improved therapies.

Chronic kidney disease is a serious complication of MMA, developing in most mut and $c b l B$ patients by the age of 8 years. ${ }^{5,6}$ Although more commonly associated with vitamin B12-nonresponsive forms of MMA, patients with mut $t^{-}$and cblA subtypes can also develop renal insufficiency and ultimately require kidney transplantation. ${ }^{7,9,10}$ Although the mechanisms of renal injury in MMA have not been elucidated, the few reported kidney biopsies have consistently demonstrated tubular injury and interstitial fibrosis with secondary glomerulosclerosis. ${ }^{11-14}$ The primary pathological manifestation of kidney injury in MMA may be mediated through mitochondrial dysfunction in the proximal renal tubule, as has been described in a Mut mouse model. ${ }^{15}$

The most common marker of renal function, serum creatinine, correlates poorly with glomerular function in patients with MMA. Walter et al. ${ }^{13}$ documented that some MMA patients had a markedly depressed glomerular filtration rate (GFR) in the setting of normal serum concentrations of creatinine. Cystatin C-based estimates of GFR, which are less dependent on muscle mass, have not yet been evaluated in the MMA population. ${ }^{16-20}$ Although the direct measurement of glomerular filtration function with agents such as inulin and iohexol is more accurate than GFR estimates, such testing is not routinely performed in MMA patients, even though a small study conducted in the

${ }^{1}$ Genetics and Molecular Biology Branch, National Human Genome Research Institute, National Institutes of Health, Bethesda, Maryland, USA; ${ }^{2}$ Kidney Disease Section, National Institute of Diabetes and Digestive and Kidney Diseases, National Institutes of Health, Bethesda, Maryland, USA. Correspondence: Charles P. Venditti (venditti@mail.nih.gov) 
late 1990s documented reduced Cr-EDTA-based GFR measurements in very young children with MMA. Although these authors did not describe other biochemical, imaging, or enzymatic studies in the patient cohort, their observations highlight the need to develop better disease-associated biomarkers and to assess the utility of noninvasive imaging techniques to monitor and predict kidney growth in affected children. ${ }^{7}$

Renal size has been proven to be a valuable and useful parameter in evaluating renal growth in normal populations and those with genetic disorders. ${ }^{21,22}$ Furthermore, studies in healthy controls and patients with kidney disease have repeatedly documented the correlation between renal length and other markers of renal size, such as renal volume and GFR. ${ }^{23-25}$ Using ultrasonographic measurements derived from a large single-center cohort of patients with MMA, we examined kidney growth and assessed the contribution of selected clinical and laboratory variables to renal size using regression analyses. The predictive equations and kidney size nomograms we have developed should provide a clinical tool to help define parameters associated with disease progression in MMA, afford prognostication for long-term management, and help assess renoprotective interventions in the future.

\section{Clinical studies}

\section{MATERIALS AND METHODS}

Clinical and laboratory investigations were conducted through National Institutes of Health study 04-HG-0127, titled "Clinical and Basic Investigations of Methylmalonic Acidemia and Related Disorders" (clinicaltrials.gov identifier: NCT00078078) in compliance with the Declaration of Helsinki. All subjects or their guardians gave informed assent or consent. Subjects were evaluated during the period 2004-2011. Analysis included 84 renal ultrasound studies, each imaging the right and the left kidneys, from 50 nontransplanted patients aged 2.2-36.3 years with enzymatic and mutation-confirmed forms of MMA. We treated each ultrasound from subjects with consecutive studies as a separate data point instead of averaging each patient's data because of the significant time interval, typically longer than 1 year, between visits. Patients were excluded after they had undergone a kidney or combined liver-kidney transplantation. The MMA patients were compared with a published control group of 209 patients from US medical centers who were aged 0-19 years with indications for ultrasound that included urinary tract infections, enuresis, aniridia, scoliosis, and undescended testicle; this approach has been used previously. ${ }^{26}$

\section{Clinical and laboratory measurements}

Data included height, weight, and age, as well as concentrations of serum creatinine, serum methylmalonic acid, cystatin $\mathrm{C}$, and urine methylmalonic acid. All laboratory testing was performed by standard measures through either the Department of Laboratory Medicine at the National Institutes of Health or Mayo Medical Laboratories. Patients were subdivided into mut, $c b l A$, and $c b l B$ groups on the basis of cellular complementation and molecular testing. Body surface area (BSA) was calculated using the formula ${ }^{27}$ BSA $\left(\mathrm{m}^{2}\right)=([$ height $(\mathrm{cm})$ $\times$ weight $(\mathrm{kg})] / 3,600)^{1 / 2}$. Estimated GFR (eGFR) for children younger than 18 years was calculated using updated Schwartz equations that include serum creatinine, or serum creatinine and cystatin C. ${ }^{16}$ For adults, the creatinine and creatininecystatin C eGFR equations from the Chronic Kidney Disease Epidemiology Collaboration were used. ${ }^{17}$

\section{Ultrasonography and laboratory measurements}

The Philips iU22 xMATRIX Ultrasound System (Philips, Andover, MA) with the C5-1 1-5 MHz bandwidth transducer and the General Electric LOGIQ E9 (GE, Wauwatosa, WI) with the C-1-5-D 1-5 MHz bandwidth transducer were used to obtain abdominal ultrasound studies for all study participants. Renal length was measured in the longitudinal axis. The original measurements were made in the context of research and read by one of three radiologists.

\section{Statistical analyses}

Demographic variables included age, genotype, and sex. The paired $t$-test was used to compare right and left kidney lengths. Pearson's correlation was used to determine the variation between independent variables. Published data from agematched controls ${ }^{26}$ were compared with MMA study patients using a two-sample $t$-test of the means assuming unequal variances and by a linear regression model that included the average of the right and left kidneys as the dependent variable and age as the independent variable.

In addition to variation with age, we examined the correlation of renal length with height because MMA patients can be shorter than healthy individuals. A second linear regression model was created with height as the independent variable. This linear model was compared with a different control group that created a nomogram of right and left renal length versus height using measurements from 325 German children aged 3 days to 16 years without known kidney disease. ${ }^{28}$

Using renal length as the dependent variable, the following independent variables were included in a multiple-regression equation: age, height, weight, BSA, body mass index, serum methylmalonic acid $\left(\mathrm{MMA}_{\mathrm{S}}\right)$, urine methylmalonic acid $\left(\mathrm{MMA}_{U}\right)$, serum creatinine, cystatin C, eGFR creatinine (creatinine based), eGFR creatinine-cystatin C (creatinine and cystatin $\mathrm{C}$ based), sex, and enzymatic subtype (complementation class).

The best model, as determined by the highest correlation coefficient $\left(R^{2}\right)$, was developed using stepwise regression analyses. Individual predictor variables were eliminated if the regression coefficient $(\beta)$ had a statistical significance of $P>0.05$ using the Wald test. Beta weights were calculated to assess the unique contribution of independent variables relative to the regression model (beta weight is the change in renal length when an independent variable increases $1 \mathrm{SD}$ while other independent variables are held constant). Stata version 11 (StataCorp LP, College Station, TX) was used for data manipulation and analysis.

When two or more variables were closely correlated (multicollinearity), such as $\mathrm{MMA}_{\mathrm{S}}$ and serum creatinine (Pearson 
coefficient of correlation of $0.808(P<0.0001)$; Supplementary Table S1 online), they were not included in same multipleregression model. ${ }^{29}$ Multicollinear relationships were observed between the variable group of creatinine, cystatin C, and $\mathrm{MMA}_{\mathrm{S}}$, and when controlling for height, the $R^{2}$ values for these predictors were similar (Supplementary Table $\mathbf{2}$ online). Height, BSA, and weight also demonstrated multicollinearity when controlling for height, with $R^{2}$ values of $0.767,0.757$, and 0.712 , respectively.

\section{RESULTS}

\section{Demographics and MMA subtypes}

Ultrasound measurements were available for 58 patients with isolated MMA who participated in our study. The average age of patients was 11.9 years, and the age range was 2.2-36.3 years. After excluding seven patients who had kidney transplants and one patient with distorted renal anatomy due to severe scoliosis, 50 patients $(86.2 \%)$ were evaluated for further study. Of these patients, 35 were mut subtype (yielding 63 ultrasound studies), 9 cblA subtype (14 ultrasound studies), and 6 cblB subtype (7 studies). A single kidney study included an ultrasound of both right and left kidneys. Eighteen patients had sequential ultrasound studies, yielding an additional 33 kidney studies.

\section{Differences in renal length by anatomy, subtype, and sex}

In the entire study group, the mean left kidney length was $0.37 \mathrm{~cm}$ greater than that of the right $(P<0.001)$; similar differences were noted in studies of healthy children. ${ }^{30}$ On the basis of these findings, the data were divided into those for left and right kidneys for subsequent multiple-regression analyses. The mut patients had slightly but not significantly smaller kidneys on average as compared with the $c b l A$ and $c b l B$ subtypes, and the enzymatic subtype was insignificant when added into the regression analysis (controlling for height and cystatin $\mathrm{C}$ ): $m u t$ $(P=0.487)$, cblA $(P=0.208)$, and $c b l B(P=0.485)$. Therefore, renal data from all subtypes were pooled. Likewise, sex was not a significant variable $(P=0.554)$.

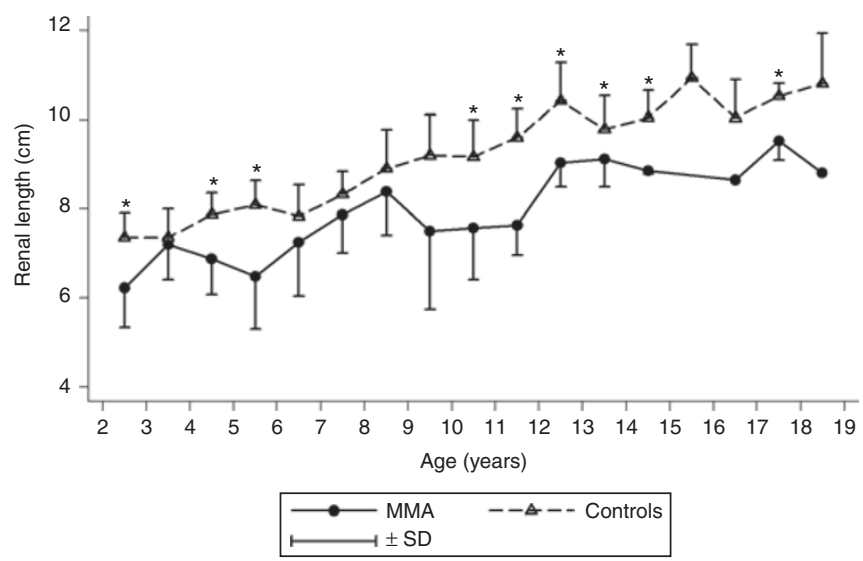

Figure 1 Renal length (average of left and right) versus age in patients with methylmalonic acidemia (MMA) and age-matched controls. ${ }^{26}$ Mean \pm SD. ${ }^{*} P<0.05$.

\section{Renal length: correlations with age and height}

Figure 2a,b consists of nomograms showing renal length variation with age and height, respectively. For more severely affected patients, with serum methylmalonic acid levels $>2,000$ $\mu \mathrm{mol} / \mathrm{l}$, renal lengths were smaller across the age and height spectrum (Figure 2).

When comparing with a control group, MMA patients had a significant difference in average renal length $(P<0.05)$, as shown in Table 1 and Figure $1 .^{26}$ The regression equation for the control group was renal length $(\mathrm{cm})=6.79+0.22 \times$ age (years) for children older than 1 year. The linear regression equation for MMA subjects was $6.80+0.09 \times$ age (years) with $P<0.001$ for both the constant and $\beta_{\text {age }}$ coefficient. In addition to age $\left(R^{2}=0.40\right)$, renal length varied linearly with height $\left(R^{2}=\right.$ $0.51)$ (Figure $2 \mathrm{~b})$ and BSA $\left(R^{2}=0.52\right)$. Because the published controls averaged right and left kidney lengths when developing their nomogram, we used an average of the right and left kidneys for comparison. ${ }^{26}$
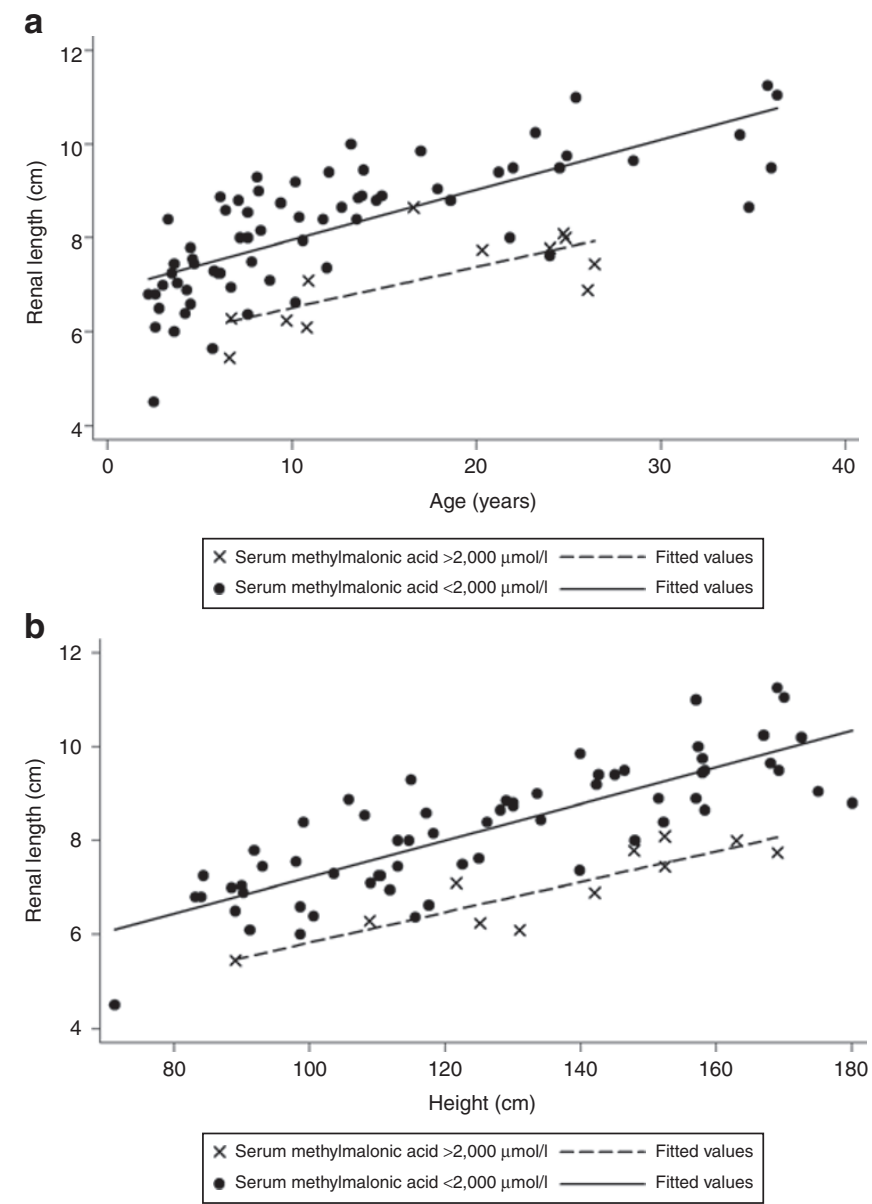

Figure 2 Renal length variation with age and height. (a) Renal length versus age, stratified by serum methylmalonic acid level. Patients with $>2,000 \mu \mathrm{mol} / \mathrm{l}$ serum methylmalonic acid have smaller kidneys. (b) Renal length versus height, stratified by serum methylmalonic level. Similar to (a), more severely affected patients with higher serum methylmalonic acid levels have smaller kidneys across the height spectrum. 
Table 1 Renal length of MMA subjects versus controls, by age

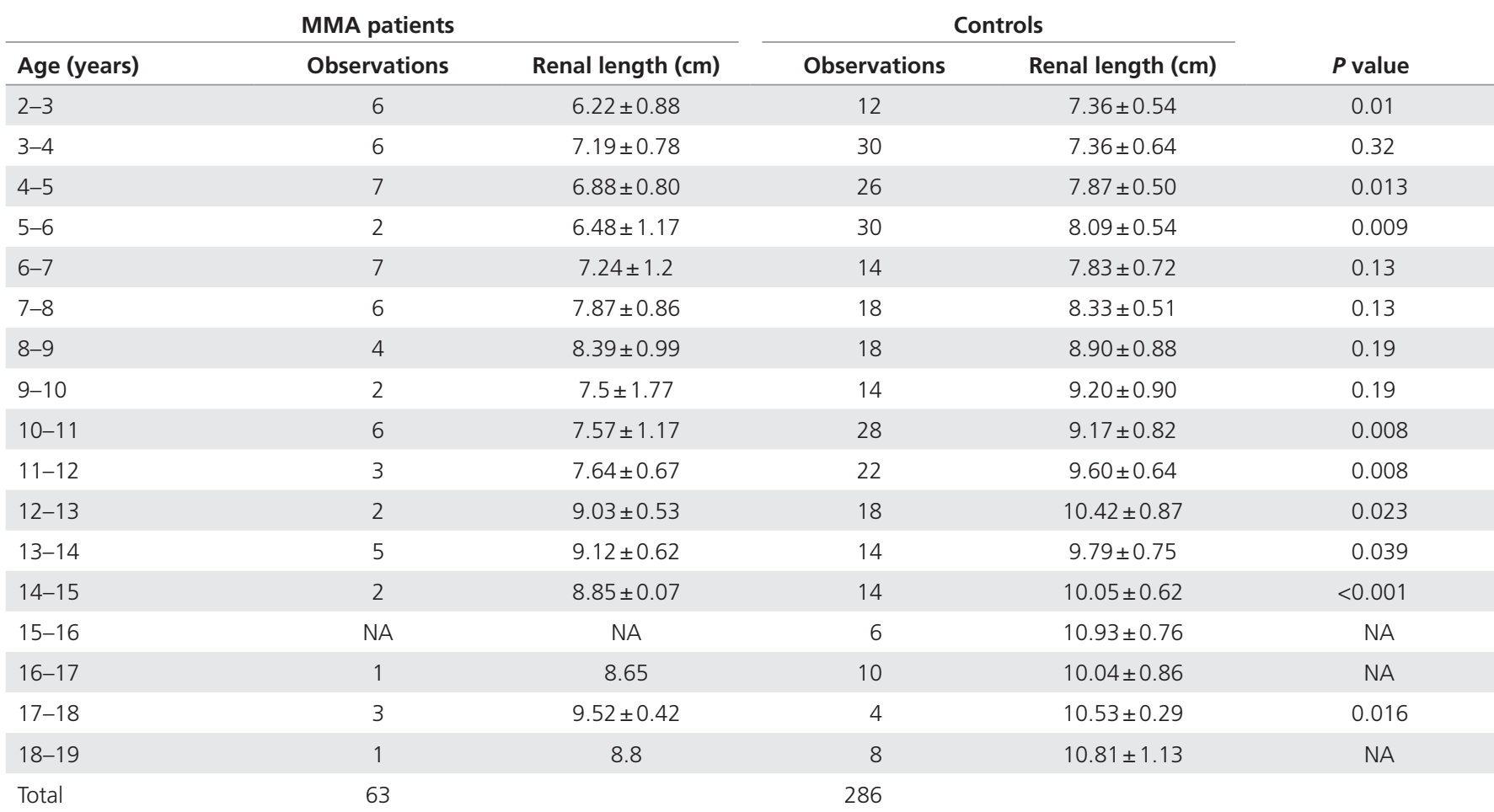

The average and SD for renal length values for MMA patients (all enzymatic subtypes) and control subjects are presented in 1 -year age intervals. Right and left renal lengths are averaged. Comparisons using the $t$-test assumed unequal variances. For controls, data are taken from Rosenbaum et al. (ref. 26).

MMA, methylmalonic acidemia; NA, not available.

With height as the independent variable and renal length as the dependent variable, MMA patients had a diminished slope as compared with historical controls, indicating that in MMA there is a decreased renal growth rate that is independent of height. ${ }^{28}$ For this group of published controls, renal length nomograms were developed for left and right kidneys separately. ${ }^{28}$ The control group equations were left kidney length $(\mathrm{cm})=0.051 \times$ height $(\mathrm{cm})+1.8$, and right kidney length $(\mathrm{cm})$ $=0.050 \times$ height $(\mathrm{cm})+1.8$. The MMA patient equations were left kidney length $(\mathrm{cm})=0.033 \times$ height $(\mathrm{cm})+4.0$, and right kidney length $(\mathrm{cm})=0.037 \times$ height $+3.0(P<0.001$ for $\beta$ coefficients).

Renal length correlations with serum and urine laboratory values

Serum creatinine, $\mathrm{MMA}_{S}$, and $\mathrm{MMA}_{\mathrm{U}}$ were analyzed according to enzymatic subtype and age (Supplementary Table S3 online). For the mut and cblA groups, serum creatinine and $\mathrm{MMA}_{\mathrm{S}}$ increased with age as compared with $\mathrm{MMA}_{\mho}$, which remained unchanged over time. The number of patients in the $c b l B$ group was too small to see trends similar to those of the $m u t$ and $c b l A$ groups. Supplementary Table $\mathbf{S 1}$ online shows that creatinine, $\mathrm{MMA}_{\mathrm{s}}$, and $\mathrm{MMA}_{\mathrm{U}}$ significantly $(P<0.05)$ correlated with age, with Pearson's correlation coefficients of $0.503,0.235$, and -0.256 , respectively, for all enzymatic subtypes. Supplementary Figure $\mathbf{S 1}$ online shows serum creatinine, $\mathrm{MMA}_{S}$, and $\mathrm{MMA}_{\mathrm{U}}$ variations with age. In more severely affected patients (those with a serum methylmalonic acid level of $\geq 2,000 \mu \mathrm{mol} / \mathrm{l}$ ), serum creatinine rises faster with age (Supplementary Figure S1a online); conversely, $\mathrm{MMA}_{\mathrm{S}}$ versus age has a greater slope when correlated with eGFR creatinine level $>60 \mathrm{ml} / \mathrm{min} / 1.73 \mathrm{~m}^{2}$ (Supplementary Figure S1b online).

Routine measurement of serum cystatin $\mathrm{C}$ began midway through the study, with 33 of the 84 ultrasounds having matched serum cystatin C values. Cystatin C (Supplementary Table S1 online) correlated closely with serum creatinine and MMAs and had Pearson correlation coefficients of 0.790 and 0.768 , respectively. We also analyzed $\mathrm{MMA}_{\mathrm{s}}$ as compared with eGFR creatinine-cystatin $\mathrm{C}$ and found that $\mathrm{MMA}_{\mathrm{s}}$ rises sharply when the eGFR creatinine-cystatin C level is $<60 \mathrm{ml} / \mathrm{min} /$ $1.73 \mathrm{~m}^{2}$ (Figure 3). Figure 4 shows patients with serum methylmalonic acid levels $\geq 2,000 \mu \mathrm{mol} / \mathrm{l}$ have lower eGFR creatinine when conrolled for age.

\section{Multiple-regression modeling}

The most predictive multiple-regression model for renal length found cystatin $\mathrm{C}$ and height to be the optimal independent variables, with beta weights of -0.51 and 0.75 , respectively. Supplementary Figure S2 online is a representation of this regression model showing that renal length is inversely proportional to cystatin $\mathrm{C}$ when stratified by height. The final predictive equation for left kidney length $(\mathrm{cm})=4.9-0.92 \times$ cystatin $\mathrm{C}+0.036 \times$ height; for the right kidney, predicted length $(\mathrm{cm})$ $=3.6-0.92 \times$ cystatin $C+0.043 \times$ height. Supplementary 


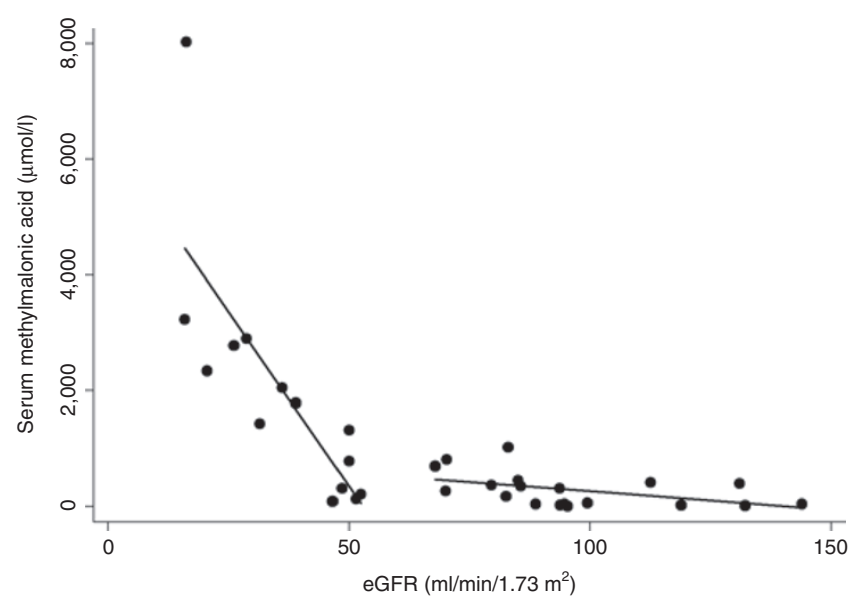

Figure 3 Serum methylmalonic acid $\left(\mathrm{MMA}_{\mathrm{s}}\right)$ for all enzymatic subtypes versus estimated glomerular filtration rate (eGFR) creatinine-cystatin C. Best fitted linear regression lines are shown for eGFR $<60$ and eGFR $>60$. $M_{M A}$ increases markedly for eGFR values below $60 \mathrm{ml} / \mathrm{min} / 1.73 \mathrm{~m}^{2}$.

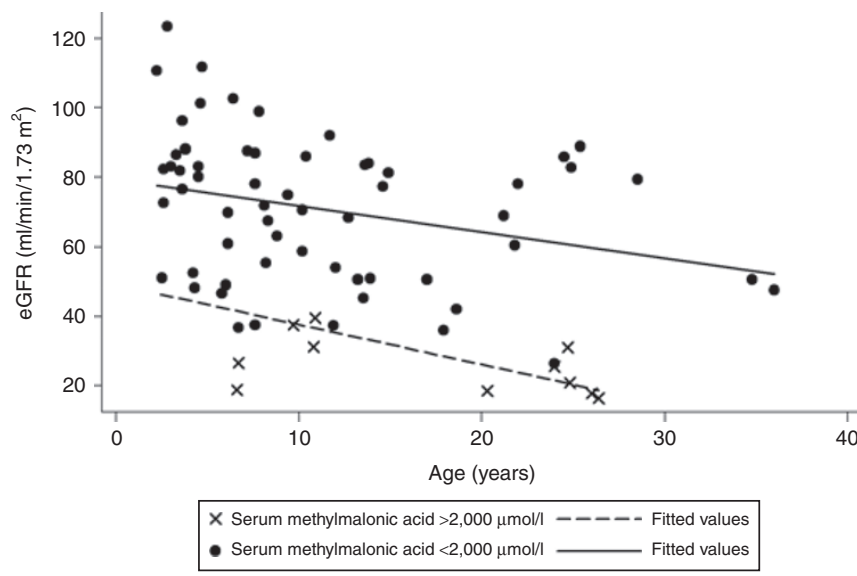

Figure 4 Estimated glomerular filtration rate (eGFR) creatinine for all enzymatic subtype patients declines with age, reflective of a progressive chronic kidney disease (CKD). eGFR calculated using the updated Schwartz equation for children (ref. 16). For adults, the creatinine equation from the Chronic Kidney Disease Epidemiology Collaboration was used (ref. 17). The solid line represents linear regression for patients with serum methylmalonic acid $<2,000 \mu \mathrm{mol} / \mathrm{l}$, and dashed lines represent a linear regression for serum methylmalonic acid $>2,000 \mu \mathrm{mol} / \mathrm{l}$. The patients with a higher serum methylmalonic acid show a lower eGFR.

Table S2 online compares $\mathrm{MMA}_{\mathrm{s}}$, cystatin C-, creatinine-, and eGFR-based multiple-regression models stratified by different creatinine levels. The regression model using $\mathrm{MMA}_{\mathrm{s}}$ was found to be highly predictive of renal length when serum creatinine level was $<1.5$ (Supplementary Table S2 online).

\section{DISCUSSION}

Chronic kidney disease due to progressive tubulointerstitial injury is emerging as a frequent manifestation in MMA and eventually results in the need for dialysis or kidney transplantation; half of our cohort of MMA patients of all ages and $100 \%$ of our $m u t^{0}$ patients older than 15 years either have been transplanted or have an eGFR $<60 \mathrm{ml} / \mathrm{min} / 1.73 \mathrm{~m}^{2}$. Kidney disease in this population is notoriously difficult to diagnose and monitor early in life because reliance on serum creatininebased eGFR estimates may overstate the true GFR due to low muscle mass, ${ }^{13}$ and kidney biopsy, the most accurate test for determining the nature and extent of renal disease, is invasive and is rarely offered to MMA patients. Once clinically manifest, renal disease in MMA patients has a relentless progression to kidney failure as is illustrated by the linear decline of eGFR in the patient population, with a faster rate of decline apparent in those with more severe metabolic parameters. The failure to detect chronic kidney disease early is certain to delay the implementation of renoprotective strategies in affected infants and children. Whereas blockade of the renin-angiotensin system is the current standard of care for adults with chronic kidney disease, experience with renoprotective measures in the pediatric population has not been as thoroughly studied. For example, there has only been one published article addressing renoprotection with renin-angiotensin system blockade in $\mathrm{MMA}^{31}$ despite the formal recognition that such studies should be pursued. ${ }^{32}$ The successful small-molecule treatments of related metabolic disorders that feature tubulointerstitial disease and progressive kidney failure, such as nephropathic cystinosis and tyrosinemia type $\mathrm{I}^{32}$ and striking preclinical efficacy of systemic adeno-associated virus gene delivery to treat the kidney disease of MMA in a mouse model provides strong practical and theoretical support for the study of renoprotective interventions in MMA patients. ${ }^{33}$

To predict renal length in MMA subjects, we first created a multivariate model and compared our data to healthy controls in the medical literature using age as the independent variable. Renal length was chosen as the dependent variable from among other ultrasongraphic measurements such as renal volume and cortical thickness because renal length has the least amount of interobserver variation and has been established to correlate with GFR. ${ }^{24,34}$ As compared with published healthy controls, most age groups in the MMA cohort had significantly smaller renal lengths. The healthy control group and the MMA group had similar constants ( $y$-intercept), 6.79 and 6.80, respectively, suggesting that kidney size was normal at birth in the MMA patients; however, the slope ( $\mathrm{cm} /$ year) of kidney length increase in the MMA group was less than half that of the controls: 0.09 vs. $0.22 \mathrm{~cm} /$ year. ${ }^{26}$ In comparison to another large study of 778 healthy children younger than 18 years, the MMA kidney growth slope was only one-third that of the controls: 0.09 vs. $0.3 \mathrm{~cm} /$ year. ${ }^{35}$ These data provide a marker of the natural history of kidney growth in MMA, one that might be used in future studies to examine the effects of renoprotective regimens and perhaps gene therapy on the renal phenotype seen in the patients.

We were also interested in the comparison of MMA patients with healthy controls when height was the independent variable, especially because MMA patients have been shown to be shorter than age-matched healthy controls., ${ }^{4,36}$ Although most of the standardized nomograms created for renal length use age as the independent variable, one study of healthy children developed a linear model with height as the independent 
variable. ${ }^{28}$ The slope of the nomogram for kidney length versus height in the MMA patients was less than in this particular control group, with right and left kidney MMA slopes of $0.037 \mathrm{~cm}$ (renal length) $/ \mathrm{cm}$ (height) and $0.033 \mathrm{~cm} / \mathrm{cm}$, respectively, and control slopes of $0.050 \mathrm{~cm} / \mathrm{cm}$ and $0.051 \mathrm{~cm} / \mathrm{cm}$, respectively. Therefore, the comparison of MMA patients to yet another set of healthy children through linear modeling when controlling for height also demonstrated that MMA patients have slower renal growth.

Using multivariate modeling, we have found that more than $70 \%$ of the variability $\left(R^{2}\right)$ in kidney length was captured by our model. To gauge how our multivariate predictive capability $\left(R^{2}\right)$ compared with those previously reported, we demonstrated a predictive capability of an independent multivariate model for a much larger study of 3,031 healthy children younger than 13 years, which found that more than $60 \%$ of variability was explained by their model $\left(R^{2}\right.$ values of 0.696 and 0.712 for right and left kidneys, respectively). ${ }^{37}$ Cystatin $\mathrm{C}$ and height were the most predictive independent variables in the multipleregression model. Our results show that cystatin $\mathrm{C}, \mathrm{MMA}_{\mathrm{s}}$, and serum creatinine had similar $R^{2}$ values when controlling for height and illustrates the significance of these variables as independent predictors of renal length.

Our results establish that for serum creatinine level $<1.5 \mathrm{mg} /$ $\mathrm{dl}, \mathrm{MMA}_{\mathrm{s}}$ is a stronger predictor of kidney length in the multivariate model than either cystatin C or eGFR creatininecystatin $\mathrm{C}$. When beta weights are included in the regression analysis, $\mathrm{MMA}_{\mathrm{s}}$ proves to be a highly significant independent variable with a value of -0.42 . The proportional relationship between $\mathrm{MMA}_{\mathrm{s}}$ and smaller renal size in this study, combined with a large beta weight for $\mathrm{MMA}_{\mathrm{s}}$, suggests that elevated $\mathrm{MMA}_{\mathrm{S}}$ is strongly associated with renal disease in methylmalonic acidemia when controlling for height by multiple regression. In addition, Figure 2a,b demonstrates smaller renal size versus age and height, respectively, in patients with a serum methylmalonic acid level $>2,000 \mu \mathrm{mol} / \mathrm{l}$. The association between $\mathrm{MMA}_{\mathrm{s}}$ and renal failure is also evident in Figure 3, where $\mathrm{MMA}_{\mathrm{S}}$ is inversely related to eGFR creatinine-cystatin $\mathrm{C}<60 \mathrm{ml} / \mathrm{min} / 1.73 \mathrm{~m}^{2}$; in addition, all of our patients with a serum methylmalonic acid level $>1,000 \mu \mathrm{mol} / \mathrm{l}$ have an eGFR creatinine $<60 \mathrm{ml} / \mathrm{min} / 1.73 \mathrm{~m}^{2}$. These observations would advocate for the routine measurement of serum methylmalonic acid concentrations in the longitudinal monitoring of patients with MMA and highlight the need for future studies to examine serum markers concomitant with a measured GFR.

To complement serum creatinine, we obtained cystatin C levels with 33 of the total 84 renal ultrasounds but added this measurement after the protocol began. Using the aggregate data, the multivariate model incorporating cystatin $\mathrm{C}$ emerged as the most precise model, explaining more than $76 \%$ of renal length variation $\left(R^{2}=0.767\right)$ when controlling for height. As expected, the regression model using cystatin $\mathrm{C}$ was more predictive than the model using GFR estimates calculated from serum creatinine and cystatin $C$ which is most likely due to muscle mass differences between MMA patients and the patients used to derive the eGFR equation. ${ }^{16,17}$ Our data suggest that serum cystatin C concentration should also be included in the routine monitoring of renal function in MMA patients and warrants further study, including validation against direct GFR assessments, especially because recent studies suggest that in the general population, GFR estimates that incorporate both serum creatinine and serum cystatin $C$ appear to be the most accurate..$^{18,19}$ In a population with reduced muscle mass such as in those with MMA, the GFR equation using cystatin $\mathrm{C}$ or cystatin $\mathrm{C}$ and MMAs may be preferred.

Prior investigations have not consistently demonstrated a relationship between $\mathrm{MMA}_{\mathrm{U}}$ and kidney disease. ${ }^{5,13} \mathrm{~A}$ multicenter, retrospective review of 35 patients suggested that MMA $_{\mathrm{u}}$ predicted chronic kidney disease as determined from calculations using the Schwartz formula; however, inter- and intraindividual variations were claimed to contribute to overlap in the enzymatic subgroups of this study. ${ }^{5}$ By contrast, Walter et al. ${ }^{13}$ found no correlation between random urinary methylmalonic acid levels and reduction in GFR. We found that the urine methylmalonic acid concentration was not a significant predictor of renal length when controlling for height $(P=0.471): \mathrm{MMA}_{\mathrm{U}}$ did not correlate with trends in serum creatinine as can be seen by the statistically insignificant Pearson's correlation $(R=0.0389)$. Given the poor correlation between $\mathrm{MMA}_{\mathrm{U}}$ and renal length or trends in serum creatinine, our data advocate using $\mathrm{MMA}_{\mathrm{S}}$ as a predictor of renal disease and including this analyte in the routine monitoring of patients with MMA.

The mut patients had slightly smaller kidneys than those of the other subgroups; however, when controlling for cystatin C and height, we did not detect a significant difference between enzymatic subtypes on kidney length. Another study found an increase in chronic kidney disease in $m u t^{0}$ and $c b l B$ as compared with other enzymatic subtypes causing isolated MMA, which would suggest that these individuals might have smaller kidneys. ${ }^{5} \mathrm{~A}$ possible explanation for this discrepancy between the studies may be derived from the small number of participants with $c b l B$, both in our study $(n=6)$ and that of Hörster et al. ${ }^{5}(n=9)$. In addition to MMA subtypes, we did not find sex to be a contributor to renal length in MMA patients. Boys' and girls' body sizes grow at different rates; however, multiple studies, including ours, have demonstrated that renal length is not significantly different between the sexes. ${ }^{30}$

Our study has a number of limitations. Despite using the largest single-center MMA patient cohort described to date, the sample size was comparatively small, and we were unable to use a discovery and replication design. Second, the majority of our data were cross-sectional, which may introduce increased variability in the nomograms as compared with data derived from a purely longitudinal design. Third, the most severely affected patients may not have been fit for travel to the National Institutes of Health, which thus underestimated our effect size.

In summary, the rate of renal growth in the MMA subjects studied here was one-half to one-third of that observed in controls..$^{26,35}$ Renal length is likewise significantly decreased 
as compared with that of normal controls and predicted by a multiple-regression model that uses the readily available clinical variables of height and cystatin $\mathrm{C}$. When serum creatinine values fall in the age-normal range, $\mathrm{MMA}_{\mathrm{S}}$ and cystatin $\mathrm{C}$ are comparable predictors of renal length, suggesting that both should be included in the routine evaluation in MMA patients. In addition to generating a clinically useful nomogram to follow kidney growth in MMA, our results capture a marker of the natural history of the renal disease of MMA and should assist with the study of therapeutic interventions designed to target kidney disease in this population.

\section{SUPPLEMENTARY MATERIAL}

Supplementary material is linked to the online version of the paper at http://www.nature.com/gim

\section{ACKNOWLEDGMENTS}

This work was supported by the intramural research programs of the National Human Genome Research Institute and National Institute of Diabetes and Digestive and Kidney Diseases, National Institutes of Health.

\section{DISCLOSURE}

The authors declare no conflict of interest.

\section{REFERENCES}

1. Manoli I, Venditti CP. Methylmalonic acidemia. 16 August 2005 (Updated 28 September 2010). In: Pagon RA, Bird TD, Dolan CR, et al. (eds). GeneReviews ${ }^{\text {TM }}$ [Internet]. University of Washington: Seattle, WA, 1993http://www.ncbi.nlm. nih.gov/books/NBK1231/.

2. Matsui SM, Mahoney MJ, Rosenberg LE. The natural history of the inherited methylmalonic acidemias. N Eng/ J Med 1983;308:857-861.

3. Nicolaides P, Leonard J, Surtees R. Neurological outcome of methylmalonic acidaemia. Arch Dis Child 1998;78:508-512.

4. van der Meer SB, Poggi F, Spada M, et al. Clinical outcome of long-term management of patients with vitamin B12-unresponsive methylmalonic acidemia. J Pediatr 1994;125(6 Pt 1):903-908.

5. Hörster F, Baumgartner MR, Viardot $C$, et al. Long-term outcome in methylmalonic acidurias is influenced by the underlying defect (mut0, mutcblA, cblB). Pediatr Res 2007;62:225-230.

6. Cosson MA, Benoist JF, Touati G, et al. Long-term outcome in methylmalonic aciduria: a series of 30 French patients. Mol Genet Metab 2009;97:172-178

7. Touati G, Valayannopoulos V, Mention K, et al. Methylmalonic and propionic acidurias: management without or with a few supplements of specific amino acid mixture. J Inherit Metab Dis 2006:29:288-298.

8. Dionisi-Vici C, Deodato F, Röschinger W, Rhead W, Wilcken B. 'Classical' organic acidurias, propionic aciduria, methylmalonic aciduria and isovaleric aciduria: long-term outcome and effects of expanded newborn screening using tandem mass spectrometry. J Inherit Metab Dis 2006;29:383-389.

9. Van Calcar SC, Harding CO, Lyne P, et al. Renal transplantation in a patient with methylmalonic acidaemia. J Inherit Metab Dis 1998;21:729-737.

10. Coman D, Huang J, McTaggart S, et al. Renal transplantation in a 14 -year-old girl with vitamin B12-responsive cblA-type methylmalonic acidaemia. Pediatr Nephrol 2006;21:270-273.

11. Molteni KH, Oberley TD, Wolff JA, Friedman AL. Progressive renal insufficiency in methylmalonic acidemia. Pediatr Nephrol 1991;5:323-326.

12. Rutledge SL, Geraghty M, Mroczek E, Rosenblatt D, Kohout E. Tubulointerstitial nephritis in methylmalonic acidemia. Pediatr Nephrol 1993;7:81-82.
13. Walter JH, Michalski A, Wilson WM, Leonard JV, Barratt TM, Dillon MJ. Chronic renal failure in methylmalonic acidaemia. Eur J Pediatr 1989;148:344-348.

14. Oberholzer VG, Levin B, Burgess EA, Young WF. Methylmalonic aciduria. An inborn error of metabolism leading to chronic metabolic acidosis. Arch Dis Child 1967:42:492-504.

15. Chandler RJ, Zerfas PM, Shanske $S$, et al. Mitochondrial dysfunction in mut methylmalonic acidemia. FASEB J 2009:23:1252-1261.

16. Schwartz GJ, Muñoz A, Schneider MF, et al. New equations to estimate GFR in children with CKD. J Am Soc Nephro/ 2009;20:629-637.

17. Inker LA, Schmid CH, Tighiouart H, et al.; CKD-EPI Investigators. Estimating glomerular filtration rate from serum creatinine and cystatin C. N Engl J Med 2012;367:20-29.

18. Bökenkamp A, Domanetzki M, Zinck R, Schumann G, Byrd D, Brodehl J. Cystatin $\mathrm{C}$ - a new marker of glomerular filtration rate in children independent of age and height. Pediatrics 1998;101:875-881.

19. Roos JF, Doust J, Tett SE, Kirkpatrick CM. Diagnostic accuracy of cystatin C compared to serum creatinine for the estimation of renal dysfunction in adults and children - a meta-analysis. Clin Biochem 2007;40:383-391.

20. Narvaez-Sanchez R, Gonzalez L, Salamanca A, et al. Cystatin C could be a replacement to serum creatinine for diagnosing and monitoring kidney function in children. Clin Biochem 2008;41:498-503.

21. Schmidt IM, Main KM, Damgaard IN, et al. Kidney growth in 717 healthy children aged 0-18 months: a longitudinal cohort study. Pediatr Nephrol 2004;19:992-1003.

22. Ortiz-Neira CL, Traubici J, Alan D, et al. Sonographic assessment of renal growth in patients with Beckwith-Wiedemann syndrome: the Beckwith-Wiedemann syndrome renal nomogram. Clinics (Sao Paulo) 2009:64:41-44.

23. Adibi A, Adibi I, Khosravi P. Do kidney sizes in ultrasonography correlate to glomerular filtration rate in healthy children? Australas Radio/ 2007;51:555-559.

24. Paleologo G, Abdelkawy H, Barsotti M, et al. Kidney dimensions at sonography are correlated with glomerular filtration rate in renal transplant recipients and in kidney donors. Transplant Proc 2007;39:1779-1781.

25. Di Zazzo G, Stringini G, Matteucci MC, Muraca M, Malena S, Emma F. Serum creatinine levels are significantly influenced by renal size in the normal pediatric population. Clin J Am Soc Nephrol 2011;6:107-113.

26. Rosenbaum DM, Korngold E, Teele RL. Sonographic assessment of renal length in normal children. AJR Am J Roentgenol 1984;142:467-469.

27. Mosteller RD. Simplified calculation of body-surface area. N Engl J Med 1987;317:1098.

28. Dinkel E, Ertel M, Dittrich M, Peters H, Berres M, Schulte-Wissermann H. Kidney size in childhood. Sonographical growth charts for kidney length and volume. Pediatr Radiol 1985;15:38-43.

29. Stoltzfus JC. Logistic regression: a brief primer. Acad Emerg Med 2011;18:10991104

30. Zerin JM, Blane CE. Sonographic assessment of renal length in children: a reappraisal. Pediatr Radiol 1994;24:101-106

31. Ha TS, Lee JS, Hong EJ. Delay of renal progression in methylmalonic acidemia using angiotensin II inhibition: a case report. J Nephrol 2008;21:793-796.

32. Foreman JW. Cystinosis and Fanconi Syndrome. In: Avner ED, Harmon W, Niaudet P (eds). Pediatric Nephrology, 5th edn. Lippincott Williams \& Wilkins: Philadelphia, 2004:789-806

33. Sénac JS, Chandler RJ, Sysol JR, Li L, Venditti CP. Gene therapy in a murine model of methylmalonic acidemia using rAAV9-mediated gene delivery. Gene Ther 2012;19:385-391.

34. Schlesinger AE, Hernandez RJ, Zerin JM, Marks TI, Kelsch RC. Interobserver and intraobserver variations in sonographic renal length measurements in children. AJR Am J Roentgenol 1991;156:1029-1032.

35. Akhavan A, Brajtbord JS, McLeod DJ, Kabarriti AE, Rosenberg HK, Stock JA. Simple, age-based formula for predicting renal length in children. Urology 2011;78:405-410.

36. Hauser NS, Manoli I, Graf JC, Sloan J, Venditti CP. Variable dietary management of methylmalonic acidemia: metabolic and energetic correlations. Am J Clin Nutr 2011:93:47-56.

37. Luk WH, Lo AX, Au-Yeung AW, et al. Renal length nomogram in Hong Kong Asian children: sonographic measurement and multivariable approach. $J$ Paediatr Child Health 2010;46:310-315. 